\title{
Superlocalization of fracton wavefunctions excited on percolating networks
}

\author{
K YAKUBO* and T NAKAYAMA \\ Department of Applied Physics, Hokkaido University, Sapporo 060, Japan
}

\begin{abstract}
The characteristics of fracton wavefunctions excited on two-dimensional sitepercolating networks are investigated using an array-processing supercomputer. It is found that fracton modes possess a quite unique character; i.e., the core of fracton excitations falls off sharply at their edges. The exponent $d \phi$, which indicates the strength of the localization of the fracton core, is much larger than unity. It is suggested that one should be careful when calculating the matrix elements relevant to the inelastic light scattering.
\end{abstract}

Keywords. Fracton wavefunctions; site-percolating networks; array-processing supercomputer; fracton excitations; inelastic light-scattering.

The vibrational properties of percolating networks have received considerable interest in recent years (Alexander and Orbach 1982; Rammal and Toulouse 1983; Alexander 1986; Orbach 1986). The density of states (DOS) of percolating clusters in all Euclidean dimensions $d(d \geqslant 2)$ obeys the universal law, $D(\omega) \sim \omega^{1 / 3}$, in the regime above the characteristic frequency $\omega_{c}$ corresponding to the wavelength equal to the correlation length $\xi_{p}$. Rammal and Toulouse (1983) have suggested that fractons are localized as far as $\bar{d} \leqslant 2$. In this connection, Alexander et al (1986) have proposed that the ensemble average of vibrational fracton-wavefunctions takes the form,

$$
\left\langle\phi_{f r}\right\rangle \sim \exp \left[-(r / \Lambda(\omega))^{d \phi}\right]
$$

where an unimportant prefactor relevant to a long distance far from the origin $(r=0)$ is not taken into account. $\Lambda(\omega)$ is the characteristic length of the fracton-mode. The exponent $d \phi$ is a measure of the strength of the localization. The excitations with $d \phi$ larger than unity are called superlocalized modes (Levy and Souillard 1987). Theoretical predictions for the magnitude of the exponent $d \phi$, however, are controversial at present (Harris and Aharony 1987; Levy and Souillard 1987).

This report investigates, using a supercomputer, the nature of localized fracton excitations on two-dimensional (2D) percolating clusters. We concentrate on the shape of the ensemble-averaged fracton. It is shown that the amplitude of a single fracton falls off sharply at their edges. This sharp fall-off is clearly a consequence of the motion of reasonably large numbers of atoms moving together independently of the motion of the remainder of atoms in the network. We call this portion the core of fracton-eigenmode.

Consider a site-percolating cluster consistng of $N$ atoms with mass $m$ and linear

\footnotetext{
* For correspondence
} 
springs connecting two nearest-neighbor atoms. The equations of motion of this system are simple and are expressed by

$$
m \ddot{u}_{i}(t)-\sum_{j} K_{i j} u_{j}(t)=0,
$$

where $u_{i}$ is the scalar displacement of the atom with mass $m$ on the $i$ th site. The force constant is taken to be $K_{i j}=0(i \neq j)$ if either site $i$ or $j$ is unoccupied, and $K_{i j}=1$ otherwise. Diagonal elements satisfy the following relation $K_{i i}=-z_{i}$ where $z_{i}$ is the coordination number of the site $i$. It should be noted here, that if we put $K_{i i}=0$, our system is transferred to the quantum percolation problem.

In standard numerical methods, the dynamical matrix $\left[K_{i j}\right]$ is diagonalized directly to obtain the eigenfrequencies and eigenmodes of the system described by (2). These methods, however, require a large amount of computer memory space (of the order of $N^{2}$ ) and relatively long CPU time. Due to these conditions, the site numbers we can treat are limited in general to within a few thousands. In addition, these standard numerical routines yield poor accuracy for the low-frequency eigenvalues and eigenvectors. The method employed in the present work enables us to treat the eigenvalue problem of very large systems, as large as $N>10^{5}$, because the algorithm requires less memory space (of the order of $N$ ) and is extraordinarily suitable for array-processing supercomputers. The method is based on the physical analogy that the eigenfrequency of the system satisfies the resonance condition when applying a periodic external force with frequency $\omega$ to the system.

We have checked our algorithm of computing the shape of localized wavefunctions, by comparing the localized mode-pattern on a linear chain calculated by our method with that of the corresponding exact solution. The system was constructed of 99 atoms with unit mass, and one light impurity with mass $m=0.8$ located in the center of the linear chain, which is connected by linear springs (force constant $K=1$ ). The exact solution for the wavefunction of the localized impurity state is written as $\phi(r, t) \sim \exp \left[-(r / d)^{a}\right]$, where $a=1$ and $d=2 \cdot 466$. Our numerical result provides $a=1.005$ and $d=2 \cdot 506$. The agreement bewteen our numerical result and the exact solution can be improved by increasing the site number.

The mode-patterns of fractons were calculated by applying our method to 2D-percolating networks. Networks at $p_{c}=0.593$ were formed on $700 \times 700$ square lattices. On percolating networks at $p_{c}$ all excited modes $(\omega<1)$ should behave as fractons. The typical mode-pattern with $\omega=0.01$ is shown in figure 1 , where the displacements of atoms are shown by oblique arrows. Figure 1 shows the magnified picture of the mode-pattern excited on a percolating network at $p_{c}$ with 169,576 occupied sites. The vibrational excitation in figure 1 is evidently very sharply localized at the edges. We should particularly note that displacements of atoms in a "peninsula" (weakly connected portions in the percolating cluster) move in phase, and the vibrational amplitudes fall off sharply at their edges. This indicates that the fracton-mode consists of the free vibration of atoms relatively weakly bound to its surroundings. It is interesting to note that the tail (the portion spread upward in the figure) from a core of fracton (the block with long arrows located at the lowest position) extends over a large distance with phases in turns (phases are indicated by directions of oblique arrows). This is because the overall center of mass of the fracton must not move during vibration, and there must be compensating mass outside of the core which moves in an opposite direction.

For the purpose of taking an ensemble-average of core of fracton wavefunctions, we prepared nine 2D site-percolating networks at $p_{c}$ formed on $700 \times 700$ square 


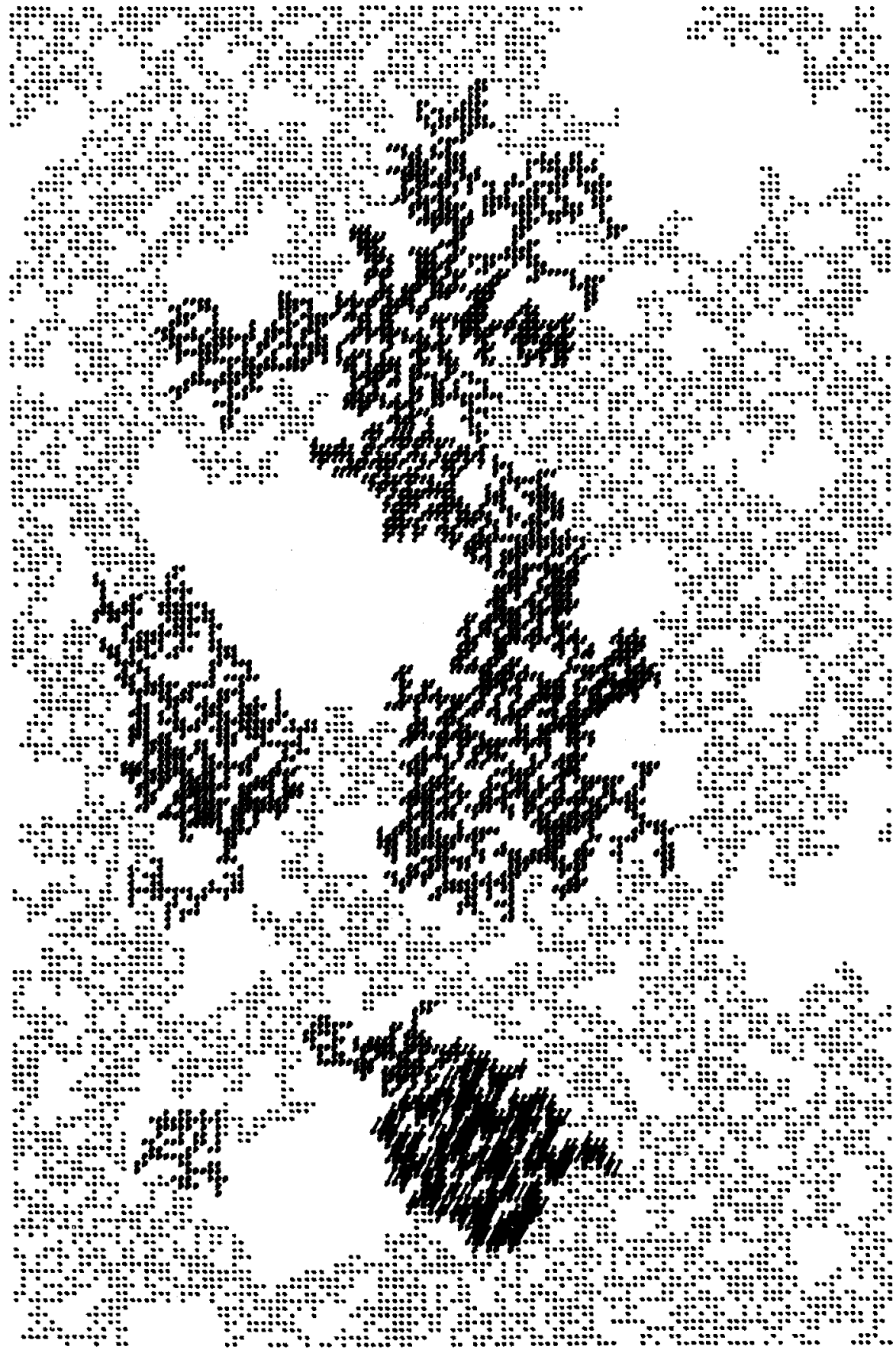

Figure 1. Magnified picture of a single fracton mode-pattern excited on a $2 \mathrm{D}$ percolating cluster $(N=169,576)$ formed on a $700 \times 700$ square lattice. Angular frequency of this mode is $n=0.01$ in units indicated in the text. The lengths of arrows indicate the amplitudes. 
lattices. The maximum cluster size was $N=171,306$ and the minimum, $N=76,665$. We excited totally 129 fracton modes with eigenfrequencies close to $\omega=0.01$ on nine percolating networks. The ensemble-averaged shape of the core of fracton wavefunction was obtained by averaging over all these fractons. We calculate the value of the exponent $d \phi$ in (1) from this averaged shape. The exponent $d \phi$ is obtained as $d \phi=2 \cdot 3 \pm 0 \cdot 1$ and the characteristic length $\Lambda(\omega=0 \cdot 01)=17 \cdot 2$.

In addition, we have calculated $d \phi$ and $\Lambda(\omega)$ for four different frequencies; $\omega=0.005$, $0.006,0.007$ and 0.008 , excited on five percolating networks. It should be emphasized that the characteristic length $\Lambda(\omega)$ clearly depends on frequency. We can find $\Lambda(\omega) \sim \omega^{-0.71}$. The scaling consideration tells us $\Lambda(\omega) \sim \omega^{-\bar{d} / D}$ (Alexander and Orbach 1982). Substituting the values $\bar{d}=4 / 3$ and $D=1.89$ for $2 D$ networks, the dispersion law should be of the form $\Lambda(\omega) \sim \omega^{-0.705}$. The fair agreement between the theory and our experiments suggests that we have surely treated fracton excitations in our ensemble.

Recently, Levy and Souillard (1987) have suggested that electronic impurity states on fractal $d \phi$ should be larger than $1+\theta / 2$ (the lower bound), where $\theta$ is the decay of the diffusion coefficient with distance. Thus, $d \phi$ should be larger than 1.43 for two-dimensional percolating cluster. Harris and Aharony (1987) have argued that the geometrical exponent $d \phi$ of typical fracton wavefunction lies in the range $1<d \phi<\zeta_{c}$, where $\zeta_{c}$ is the exponent of the typical chemical length, and takes the value 1.15 for percolation in $d=2$. Thus, the predictions on the magnitude of the geometrical exponent $d \phi$ are controversial at present, though we note that the two approaches are for electronic impurity states and not for vibrations.

The time-dependent vibrating density responsible for the inelastic scattering is expressed by

$$
\rho_{i j}(\mathbf{r}, t)=\rho_{0}(\mathbf{r}) \cdot e_{i j}(\mathbf{r}, t)
$$

where $e_{i j}(\mathbf{r}, t)$ is the local strain and $\rho_{0}(\mathbf{r})$ the static density, respectively. From the present work it is clear that the strain in percolating clusters is not uniformly distributed. The backbone stores strain energy. One should be careful when calculating the matrix element of strain, which is relevant to light scattering (Tsujimi et al 1988). Details on this work will be published in future.

\section{Acknowledgement}

The Hokkaido University Computing Center is acknowledged for the use of the computer facilities.

\section{References}

Alexander S 1986 Physica A140 397

Alexander S, Entin-Wohlman O and Orbach R 1986 Phys. Rev. B34 2726

Alexander S and Orbach R $1982 \mathrm{~J}$. Phys. (Paris) Lett. 43 L625

Harris A B and Aharony A 1987 Europhys. Lett. 41355

Levy Y-E and Souillard B 1987 Europhys. Lett. 4133

Orbach R 1986 Science 231814

Rammal R and Toulouse G 1983 J. Phys. (Paris) Lett. 44 L13

Tsujimi Y, Courtens E, Pelous J and Vacher R 1988 Phys. Rev. Lett. 602757 